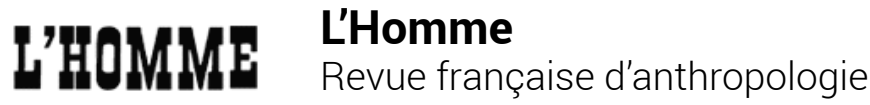

175-176 | juillet-septembre 2005

Vérités de la fiction

\section{Nathalie Heinich \& Jean-Marie Schaeffer, Art, création, fiction : entre sociologie et philosophie}

Nîmes, Jacqueline Chambon, 2004, 217 pages

\section{Corinne Delmas}

\section{(2) OpenEdition}

\section{Journals}

Édition électronique

URL : http://journals.openedition.org/lhomme/2037

DOI : 10.4000//homme.2037

ISSN : 1953-8103

\section{Éditeur}

Éditions de l'EHESS

Édition imprimée

Date de publication : 15 octobre 2005

Pagination : 475-477

ISBN : 2-7132-2035-1

ISSN : 0439-4216

\section{Référence électronique}

Corinne Delmas, « Nathalie Heinich \& Jean-Marie Schaeffer, Art, création, fiction : entre sociologie et philosophie », L'Homme [En ligne], 175-176 | juillet-septembre 2005, mis en ligne le 30 novembre 2006, consulté le 22 septembre 2020. URL : http://journals.openedition.org//homme/2037 ; DOI : https:// doi.org/10.4000//homme.2037

Ce document a été généré automatiquement le 22 septembre 2020

(C) École des hautes études en sciences sociales 


\section{Nathalie Heinich \& Jean-Marie Schaeffer, Art, création, fiction : entre sociologie et philosophie}

Nîmes, Jacqueline Chambon, 2004, 217 pages

\section{Corinne Delmas}

\section{RÉFÉRENCE}

Nathalie Heinich \& Jean-Marie Schaeffer, Art, création, fiction : entre sociologie et philosophie, Nîmes, Jacqueline Chambon, 2004, 217 p., notes bibliogr. (« Rayon art »).

1 CET OUVRAGE est original par sa démarche : croisant les regards d'une sociologue et d'un philosophe, il est constitué d'articles de Nathalie Heinich commentés par Jean-Marie Schaeffer et, en miroir, d'articles de Jean-Marie Schaeffer commentés par Nathalie Heinich. Le tout se répartit dans les trois chapitres qui composent le livre: l'art, la création et la fiction. Or, faire dialoguer philosophe et sociologue est bien une démarche originale dans un contexte marqué par la spécialisation et par un durcissement des clivages disciplinaires, nonobstant les nombreux appels à la transdisciplinarité et autres interdisciplinarités. Et, pour reprendre les termes programmatiques utilisés par Nathalie Heinich dans un précédent ouvrage : «L'interdisciplinarité n'est plus alors un œcuménisme ou un syncrétisme mou, visant à pacifier les rapports entre voisins, mais un passage de relais: le sociologue dit ce que lui autorisent ses problématiques, ses méthodes théoriques, jusqu'au point où ils ne permettent pas de dire quoi que ce soit de spécifique ou de nouveau sur un objet, qu'une discipline voisine est mieux équipée pour traiter $»^{1}$. Un tel « passage de relais » permet ici d'approfondir quelques questions centrales touchant à la création artistique et à la fiction : les frontières de l'art contemporain, les différenciations génériques, la généalogie de la figure moderne de l'artiste, la notion d'événement artistique, le statut du témoignage entre autobiographie et roman, ou encore les rapports entre fiction et 
croyance. Et l'on assiste au fil de la lecture à un mouvement de va-et-vient, à tous les niveaux, entre l'approche philosophique et l'approche sociologique, entre l'objet et la démarche également. Ainsi, les textes de Nathalie Heinich montrent-ils, dans la continuité d'autres textes de cet auteur, «ce que l'art fait à la sociologie» (p. 121), i.e. combien les valeurs artistiques ne sont ni totalement absolues ni totalement arbitraires, et combien ce domaine de recherche est à la fois réfractaire à tout essentialisme comme à tout constructivisme. Il ne peut que susciter une sociologie antiréductionniste, a-critique (la sociologie du dévoilement rendant impensables les effets pervers de ses propres dénonciations, dont le redoublement de la déprivation objective par la culpabilisation), pluraliste, descriptive, relativement neutre, soucieuse ici «d'arracher son objet au poids de la tradition esthète qui en eut longtemps le monopole ", mais aussi au sociologisme ${ }^{2}$.

2 À travers l'étude des frontières catégorielles de l'art contemporain, Nathalie Heinich se pose la question plus générale du statut des catégorisations sociales. Elle montre ailleurs, dans une perspective proche de celle d'un Erving Goffman, l'intérêt heuristique d'une démarche partant non des œuvres elles-mêmes mais des "cadres " qui en organisent la production et la circulation, et de la promotion d'une approche « situationnelle [...] consistant à partir de l'expérience partagée par les acteurs dans la pratique de production et de réception d'une œuvre» (pp. 65-66), qu'il s'agisse d'une approche « pragmatique » fondée sur l'observation empirique d'interactions concrètes, ou "institutionnelle», fondée sur l'explicitation des cadres de l'expérience, des structures et systèmes. Dans son texte, «Deux facteurs institutionnels de la différenciation générique»(pp.37-57), Jean-Marie Schaeffer reprend cette seconde approche dans une perspective comparative: d'une part, la régulation interne de la littérature chinoise ou japonaise se caractérisant par une interaction directe et, en conséquence, un nombre restreint de genres lyriques par rapport à la littérature occidentale, française par exemple, qui elle se réfère à un système littéraire, et se caractérise donc par une forte différenciation générique; d'autre part, la relation d'un système littéraire à la société globale, en opposant le drame élisabéthain, se développant dans un "réseau ouvert ", à la tragédie française qui, se déployant dans un réseau fermé, se définit par une dispersion thématique moins grande, un resserrement sur une unité de ton, une action fortement hypotaxique, un resserrement lexical et syntaxique.

3 Nathalie Heinich et Jean-Marie Schaeffer reviennent également sur la nécessité pour la sociologie de s'intéresser à la catégorisation elle-même dans une perspective antiréductionniste et pluraliste, à propos de la notion d'« événement artistique ", terme apparemment descriptif mais à la visée éminemment normative. Les questions de la singularité et de l'originalité font également l'objet d'un article stimulant de JeanMarie Schaeffer sur la "généalogie de la figure moderne de l'artiste» (pp.71-98, commenté par Nathalie Heinich, pp. 99-104). Partant des débats actuels pour ou contre l'art contemporain, concentrés autour des manifestations, ready-made et autres performances pourtant minoritaires du point de vue de la pratique artistique, l'auteur analyse les catégories d'originalité, d'invention et de génie, et leurs émergences et métamorphoses concomitantes. Ainsi serait-on passé d'une valorisation de l'original à une valorisation de l'originalité, qui, telle que nous la connaissons, ne devient dominante qu'au XVIII siècle. L'originalité, en tant que catégorie culturelle, recouvre deux significations : celle de singularité de l'œuvre et celle de nouveauté au sens de 
détachement par rapport à la tradition. On passerait ainsi de l'opposition entre authenticité et inauthenticité à l'opposition entre invention et imitation, l'œuvre étant dorénavant valorisée si elle est à l'origine d'une école ou d'une manière de faire nouvelle, et dévalorisée si elle se situe dans l'imitation stylistique. À ces dimensions de l'originalité s'ajoute, à l'époque romantique, l'idée de souveraineté de l'artiste, qui résulterait d'un glissement de la théologie chrétienne vers le domaine de la création ${ }^{3}$.

$\mathrm{Au}$ terme de ce travail de décomposition sémantique et de cette étude historique comme le souligne Nathalie Heinich -, plusieurs présupposés, tels que la croyance en un essor conjoint de l'esthétique et de la subjectivité ou encore l'idée selon laquelle la revendication d'originalité s'oppose à la tradition, sont donc remis en cause. Le même souci de promouvoir une approche pluraliste et non normative domine également dans les textes consacrés au témoignage et à la fiction, sur lesquels s'achève l'ouvrage. Les auteurs abordent les statuts de la fiction et du témoignage, et le problème plus large des représentations, à partir de questions telles que la suspicion s'attachant à tout traitement fictionnel d'expériences extrêmes de nature collective comme les camps de concentration (voir l'article de Nathalie Heinich, pp.135-151) ou telles que l'étonnement devant la tendance à mettre en avant la relation entre fiction et "vérité ». Ils remettent ainsi en cause l'opposition entre fiction et histoire (au sens de réalité), et montrent tout l'intérêt de prendre en compte le sens commun et le statut accordé à leurs fictions par des acteurs moins «naïfs " que ce que tend à voir en général le sociologue : la «manière dont les acteurs sociaux vivent leur rapport à la fiction conçue comme pratique de lecture et d'écriture enchâssée dans la vie vécue » (p. 157).

\section{NOTES}

1. . Nathalie Heinich, Ce que l'art fait à la sociologie, Paris, Minuit, 1998: 65.

2. .Cf. Nathalie Heinich, La Sociologie de l'art, Paris, La Découverte, 2004 [2001], cité p. 100; et Ce que l'art..., op. cit.

3. .Et l'analyse est proche ici, comme le souligne Nathalie Heinich dans son commentaire, de celle faite par Paul Bénichou, pour le domaine littéraire, dans Le Sacre de l'écrivain, 1750-1830: essai sur l'avènement d'un pouvoir spirituel laïque dans la France moderne (Paris, José Corti, 1985). 